\title{
Cutaneous Leishmaniasis in Saudi Arabia: A Comprehensive Overview
}

\author{
Abuzaid A. Abuzaid, ${ }^{1}$ Abdalmohsin M. Abdoon, ${ }^{1}$ Mohamed A. Aldahan,, Abdullah G. Alzahrani, ${ }^{1}$ \\ Raaft F. Alhakeem, Abdullah M. Asiri, ${ }^{1}$ Mohamed H. Alzahrani,, and Ziad A. Memish ${ }^{1-3}$
}

\begin{abstract}
Despite the great efforts by health authorities in Kingdom of Saudi Arabia (KSA), Cutaneous leishmaniasis (CL) continues to be a major public health problem in the country. Many risk factors make KSA prone to outbreaks and epidemics; among these, rapid urbanization and the huge population movement are the most important. The disease is endemic in many parts of KSA, with the majority of cases concentrated in six regions, including Al-Qaseem, Riyadh, Al-Hassa, Aseer, Ha'il, and Al-Madinah. Leishmania major (L. major) and Leishmania tropica (L. tropica) are the main dermotropic species, and Phlebotomus papatasi (vector of $L$. major) and Phlebotomus sergenti (vector of L. tropica) are the proved vectors of the disease. Psammomys obesus and Meriones libycus have been defined as the principal reservoir hosts of zoonotic CL in Al-Hassa oasis, Al-Madinah, and Al-Qaseem provinces. Clinically, males are affected more than females, and there is no variation between the Saudis and expatriates in terms of number of reported cases, but the disease tends to run a more severe course among non-Saudis. Face is the most commonly affected site, and ulcerative pattern accounts for $90 \%$ of lesions. Despite local and international recommendations of using laboratory diagnostics to confirm CL cases, most cases in KSA are diagnosed and treated on clinical grounds and local epidemiology. However, systemic parenteral sodium stibogluconate (SSG) is the first line of therapy and used to treat all CL patients irrespective of their clinical presentation or the incriminated species. In brief, more efforts are needed to combat this disease. Several aspects of the disease require more evaluation through encouragement of national and regional studies. Development of evidence based national diagnostic and management guidelines, as well as algorithms, is urgently needed to improve the practice of diagnosing and treating CL in KSA.
\end{abstract}

Keywords: clinical picture, Cutaneous leishmaniasis, diagnosis, epidemiology, Saudi Arabia, treatment

\section{Introduction}

$\mathrm{C}$ UTANeOUS leishmaniasis (CL) Is the most common form of leishmaniasis; a disease with varying clinical presentation. CL is a mild dermal condition usually self-healing, but the resulting scaring could be extremely disfiguring leading to social and psychological stigma. In general, leishmaniasis refers to a group of syndromes caused by intracellular protozoa belonging to the genus Leishmania and transmitted through the bite of blood sucking female sand fly species. It is a neglected disease affecting mostly people of poor communities in developing countries, a key reason for lack of interest of pharmaceutical industries to invest in this disease (Alvar et al. 2006, WHO 2010). Approximately, 0.7 to 1.2 million new cases of CL occur each year worldwide, with about one third of cases occurring in each of the following three regions: the Americas, the Mediterranean basin, and the western Asia from Middle East to central Asia (Alvar et al. 2012). Currently CL is endemic in 87 countries worldwide (WHO EMRO 2014).

CL was described in Kingdom of Saudi Arabia (KSA), for the first time, by Morsy and Shoura (1976) in the mid-seventies of the last century. Since that time, KSA has been contributing

\footnotetext{
${ }^{1}$ Infectious Diseases Control Directorate, Ministry of Health, Riyadh, Kingdom of Saudi Arabia.

${ }^{2}$ College of Medicine, Alfaisal University, Riyadh, Kingdom of Saudi Arabia.

${ }^{3}$ Hubert Department of Global Health, Rollins School of Public health, Emory University, Atlanta, Georgia.
}

(c) Abuzaid A. Abuzaid et al. 2017; Published by Mary Ann Liebert, Inc. This is an Open Access article distributed under the terms of the Creative Commons Attribution License, which permits unrestricted use, distribution, and reproduction in any medium, provided the original work is properly cited. 


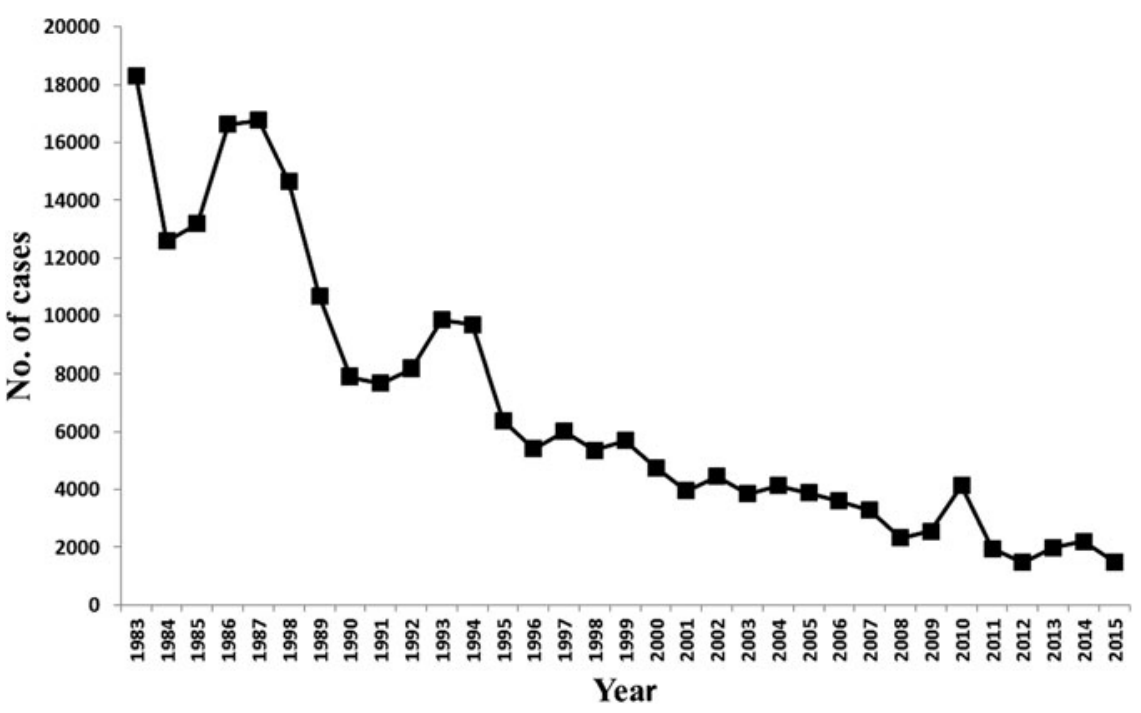

FIG. 1. Graph showing the great drop in number of reported CL cases in Saudi Arabia over the last 30 years. CL, Cutaneous leishmaniasis.

to the reported global burden of CL. KSA was among the top 10 endemic countries globally; in 1996 it was reported that $90 \%$ of CL worldwide occur in just six countries and KSA was one of these countries (Desjeux 1996). Following the establishment of the leishmaniasis control program (LCP), by the Saudi Ministry of Health (MoH) in 1978, the number of reported CL cases has dramatically dropped (Fig. 1). Currently, KSA is no longer on the list of top 10 highest endemic countries worldwide, but it is still the fourth most endemic area in western Asia (Alvar et al. 2012). Therefore, CL continues to be a major public health problem affecting the community and challenging the national health authorities. This review is meant to highlight the current situation of CL in KSA highlighting efforts made by the Saudi $\mathrm{MoH}$ and the challenges faced. The information gathered in this review was mainly obtained from the $\mathrm{MoH}$ database of monthly and annual reports and also from the online literature using the PubMed and Google search engines. A comprehensive literature search using the terms leishmaniasis and Saudi Arabia was conducted.

\section{Epidemiology}

KSA is situated in Southwest Asia with estimated land space of 2,217,949 square kilometers with different ecological and climatic patterns; it occupies $80 \%$ of the Arabian Peninsula. Several factors in KSA have led to the emergence and persistence of CL. These factors include the rapid urbanization and invasion of disease reservoir habitats by humans, the development of agricultural and irrigation schemes, the climate change, and the huge population movements, particularly, migration of nonimmune population into the endemic areas, which are known to be associated with increased transmission (WHO 2016). Climate changes affect the spatiotemporal distribution of the disease, through its impact on the survival of the vector, the developmental cycles of parasites, the movement of the host, and exposure to both types of reservoirs. Global climate change and the recent rise in temperature have led to the increase in leishmaniasis transmission overall. KSA is an open country with huge population movements; many job seekers from all over the globe come to KSA every year. In addition, millions of
Muslims arrive for Hajj pilgrimage every year and throughout the year for Umra and to visit the holy places, Makkah and Madinah.

Historically, in the year of 1973, CL has attained epidemic proportions in KSA (Al-Tawfiq and AbuKhamsin 2004, WHO EMRO 2016). Thereafter, KSA has been categorized as a highly endemic country with CL, the disease started to gain attention, and a formal registration process of cases was initiated. In 1983, a total of 18,318 new CL cases were reported (Fig. 1). This led to the development of the control program at $\mathrm{MoH}$ level. The efforts exerted by the program have resulted in dramatic decline in incidence over the subsequent years (Fig. 1). Two forms of leishmaniasis are present in KSA. In addition to CL, cases of visceral leishmaniasis (VL) are occasionally reported. Generally, sporadic cases are reported every year during the last 10 years. All cases so far have been confined to the south-western zone of KSA, mainly in Jazan and Aseer regions.

KSA is divided into 20 health regions (and administratively into 13 regions); each region reports regularly to the MoH (through monthly and annual reports) (Fig. 2). Over the past 10 years (2006-2015), the average number of cases was $\sim 2500 /$ year and ranging between 1464 and 4131 cases (Table 1). Throughout this period there were no major changes with regards to the regional incidences and distributions of reported cases except for two regions: Makkah region which used to previously report cases became a free zone in 2015 and Al-Jouf which was a free zone till the year 2013 (Fig. 3). The regions of Al-Hassa, Madinah, Ha'il, and Al-Qaseem were the most endemic regions with no changes in endemicity over this time. However, slight fluctuations in incidences have been seen in some regions (Fig. 3).

In the year 2015, a total number of 1490 new cases were formally registered (Table 1). Nevertheless, these official passive surveillance figures are likely to underestimate the disease prevalence and the true burden. Underestimation of CL occurrence is of a major concern in the field of leishmaniasis (Desjeux 2004, WHO 2007). Underreporting of patients is a big problem facing the accuracy of the official surveillance data of CL in many countries, including KSA, and it has been documented by many studies, for example, 


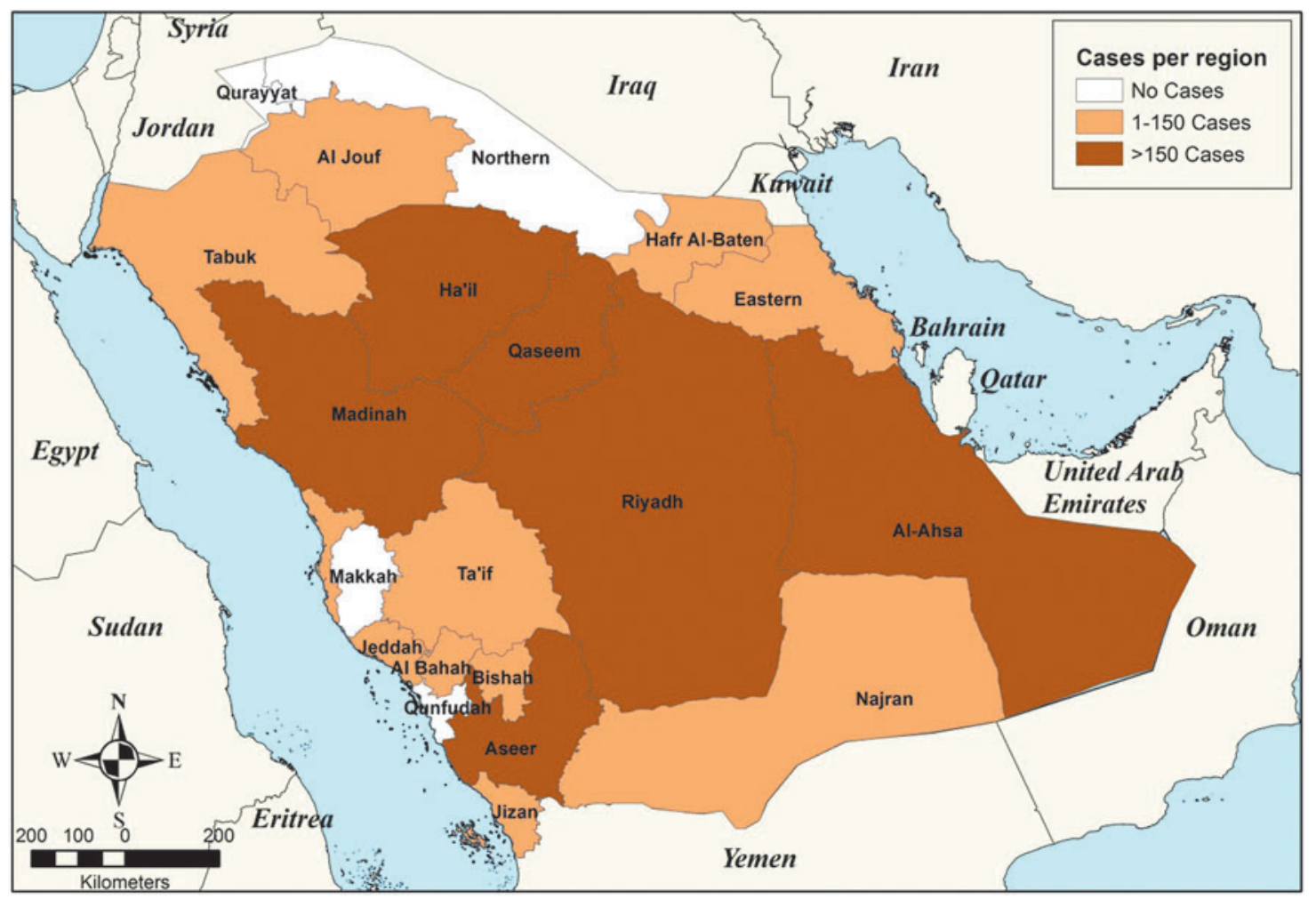

FIG. 2. Map of the Kingdom of Saudi Arabia showing the distribution of reported cases of CL in 2015 by region.

studies from Jordan and Guatemala (Dye et al. 1989, Copeland et al. 1990, Mosleh et al. 2008). The flagging interface between the private sectors and surveillance systems is one of the obstacles to gain accurate data. Moreover, the community awareness, which is a fundamental element to any infectious disease control program, may adversely affect the true incidence of CL. Knowledge of CL as a self-healing condition and the fact that scarring may occur even with treatment, combined with low income of patients, as most of them are poor people, would lead to nonreporting to

Table 1. Cases of Cutaneous Leishmaniasis Reported by Regions, from 2006 to 2015

\begin{tabular}{|c|c|c|c|c|c|c|c|c|c|c|c|}
\hline Region & 2006 & 2007 & 2008 & 2009 & 2010 & 2011 & 2012 & 2013 & 2014 & 2015 & Total \\
\hline Riyadh & 306 & 305 & 325 & 235 & 401 & 230 & 135 & 221 & 249 & 174 & 2581 \\
\hline Makkah & 10 & 9 & 11 & 3 & 6 & 1 & 3 & 3 & 4 & 0 & 50 \\
\hline Jeddah & 1 & 1 & 1 & 1 & 0 & 0 & 2 & 0 & 17 & 11 & 34 \\
\hline Ta'if & 53 & 41 & 45 & 31 & 15 & 13 & 13 & 13 & 16 & 28 & 268 \\
\hline Madinah & 643 & 619 & 287 & 626 & 1000 & 405 & 236 & 591 & 408 & 203 & 5018 \\
\hline Al-Qaseem & 981 & 851 & 758 & 654 & 1464 & 534 & 368 & 374 & 591 & 192 & 6767 \\
\hline Eastern & 54 & 14 & 18 & 10 & 11 & 11 & 10 & 22 & 30 & 21 & 201 \\
\hline Al-Hassa & 846 & 817 & 379 & 444 & 457 & 215 & 273 & 296 & 195 & 196 & 4118 \\
\hline Hafr-Al-Baten & 0 & 2 & 0 & 6 & 2 & 1 & 6 & 2 & 5 & 3 & 27 \\
\hline Aseer & 143 & 146 & 130 & 156 & 261 & 188 & 129 & 161 & 126 & 189 & 1629 \\
\hline Bishah & 2 & 2 & 7 & 25 & 10 & 16 & 10 & 10 & 0 & 3 & 85 \\
\hline Tabuk & 149 & 165 & 90 & 106 & 159 & 125 & 97 & 69 & 68 & 108 & 1136 \\
\hline Ha'il & 249 & 189 & 165 & 186 & 234 & 117 & 122 & 158 & 398 & 282 & 2100 \\
\hline Northern & 0 & 0 & 0 & 0 & 1 & 0 & 0 & 3 & 0 & 0 & \\
\hline Jizan & 46 & 51 & 63 & 28 & 81 & 75 & 31 & 39 & 15 & 7 & 436 \\
\hline Najran & 70 & 30 & 12 & 15 & 15 & 11 & 18 & 21 & 50 & 54 & \\
\hline Al-Bahah & 45 & 44 & 30 & 23 & 12 & 9 & 11 & 5 & 13 & 17 & 209 \\
\hline Al-Jouf & 1 & 0 & 0 & 0 & 0 & 0 & 0 & 0 & 5 & 2 & \\
\hline Qunfudhah & 0 & 0 & 0 & 0 & 0 & 0 & 0 & 0 & 0 & 0 & 0 \\
\hline Qurayyat & 3 & 0 & 0 & 0 & 0 & 0 & 0 & 0 & 0 & 0 & 3 \\
\hline Total & 3602 & 3286 & 2321 & 2549 & 4129 & 1951 & 1464 & 1988 & 2190 & 1490 & 24,970 \\
\hline
\end{tabular}


2007

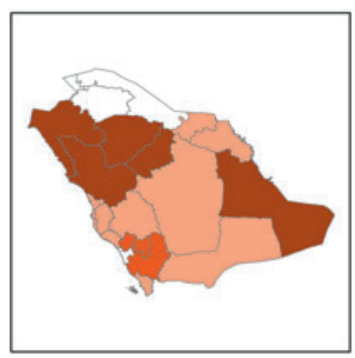

2010

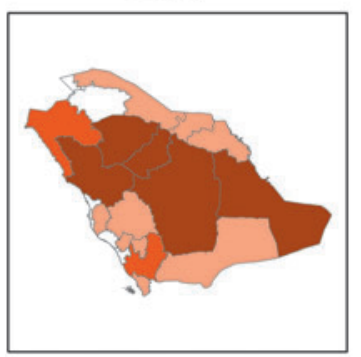

2013

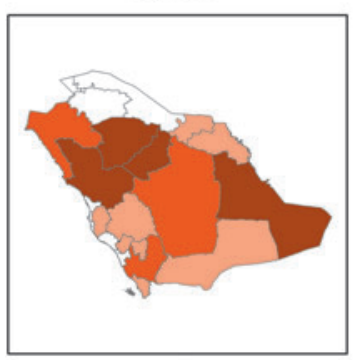

Legend

Free
2008

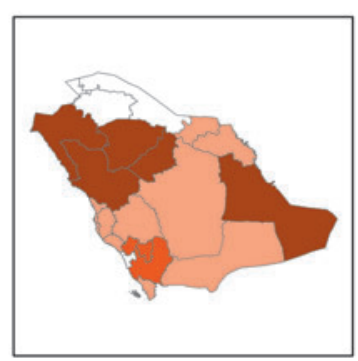

2011

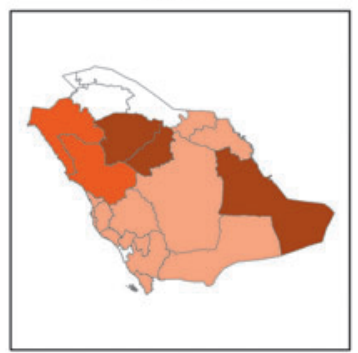

2014

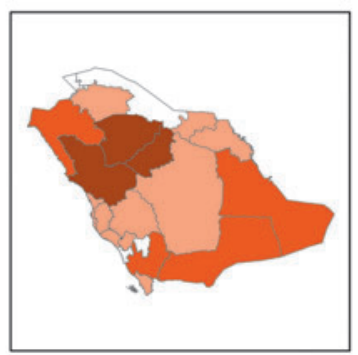

Average
2009

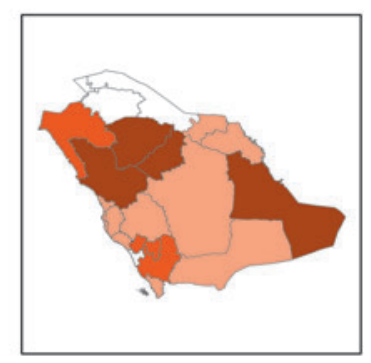

2012

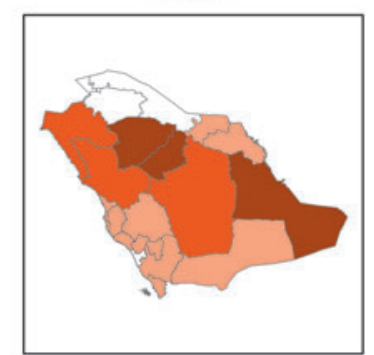

2015

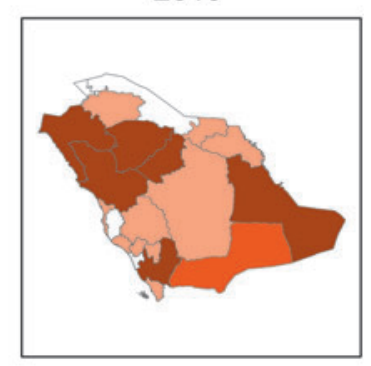

FIG. 3. Categorization of regional endemicity according to the incidence rate/100,000 population. High $(>10$ cases/100,000 population), Average (5$10 / 100,000)$, Low $(<5 / 100,000)$, and Free (no cases).

health facilities, and sometimes searching for traditional therapies. This possibility justifies the need for implementation of active case detection tools. Relying on passive case detection only is not sufficient to have a complete epidemiological data on CL.

The endemicity of the disease varies among different regions, with some regions containing hot foci of transmission and others are disease free. Typically, CL is a sporadic disease in its endemic areas and tends to attain outbreak figures when nonimmune populations visit these areas, for instance, during big construction and military operations. Few years ago in Al-Hassa region (East of the country), a great percentage of construction laborers structuring a governmental building, who were not from the area, developed the disease within a short period of time and this was, of course, accompanied by interruption of the construction operation.

Each year, the vast majority of CL cases in KSA are reported from endemic foci in the regions of Al-Qaseem and Riyadh (Central), Al-Hassa (East), Aseer (South West), and Ha'il and Al-Madinah (North West). These six regions jointly account for $83 \%$ of the total cases reported in 2015. As per the incidence data by regions for the year 2015, Ha'il is the highest endemic focus ( 282 newly detected cases), followed by Al-Madinah (203 newly detected cases) and Al-Hassa (196 newly detected cases) (Table 1, Fig. 2). The seasonality of the disease is mainly associated with winter, during which the highest numbers of cases are registered. The numbers of cases start to increase in September, remain high in the following months, and reach its peak in January and February; the number of patients then begin to decline in March and reaches its lowest number between April and July.

\section{Leishmania Parasite Endemic in KSA}

Leishmaniases are caused by more than 20 different species of the genus Leishmania (Lainson and Shaw 1987, Desjeux 1992, WHO 2010). Based on the Eurocentric world view, the different clinical forms of leishmaniasis and the incriminated Leishmania parasites are classified into old world and new world pathologies. CL in the old world, old world cutaneous leishmaniasis (OWCL), is caused mainly by three Leishmania species as follows: L. major, L. tropica, and L. aethiopica (Desjeux 1991). However, L. donovani and $L$. infantum, which are primarily incriminated in causation of VL, have been also isolated from cutaneous lesions (Pearson and Sousa 1996).

Several dermotropic species are incriminated in KSA. Isolation and parasite identification have been addressed by many studies and it has been proved that L. major (zoonotic species) and L. tropica (anthroponotic species) are the main 
FIG. 4. Geographical distribution of Zoonotic (ZCL) and Anthroponotic (ACL) cutaneous leishmaniasis based on the defined parasite species.

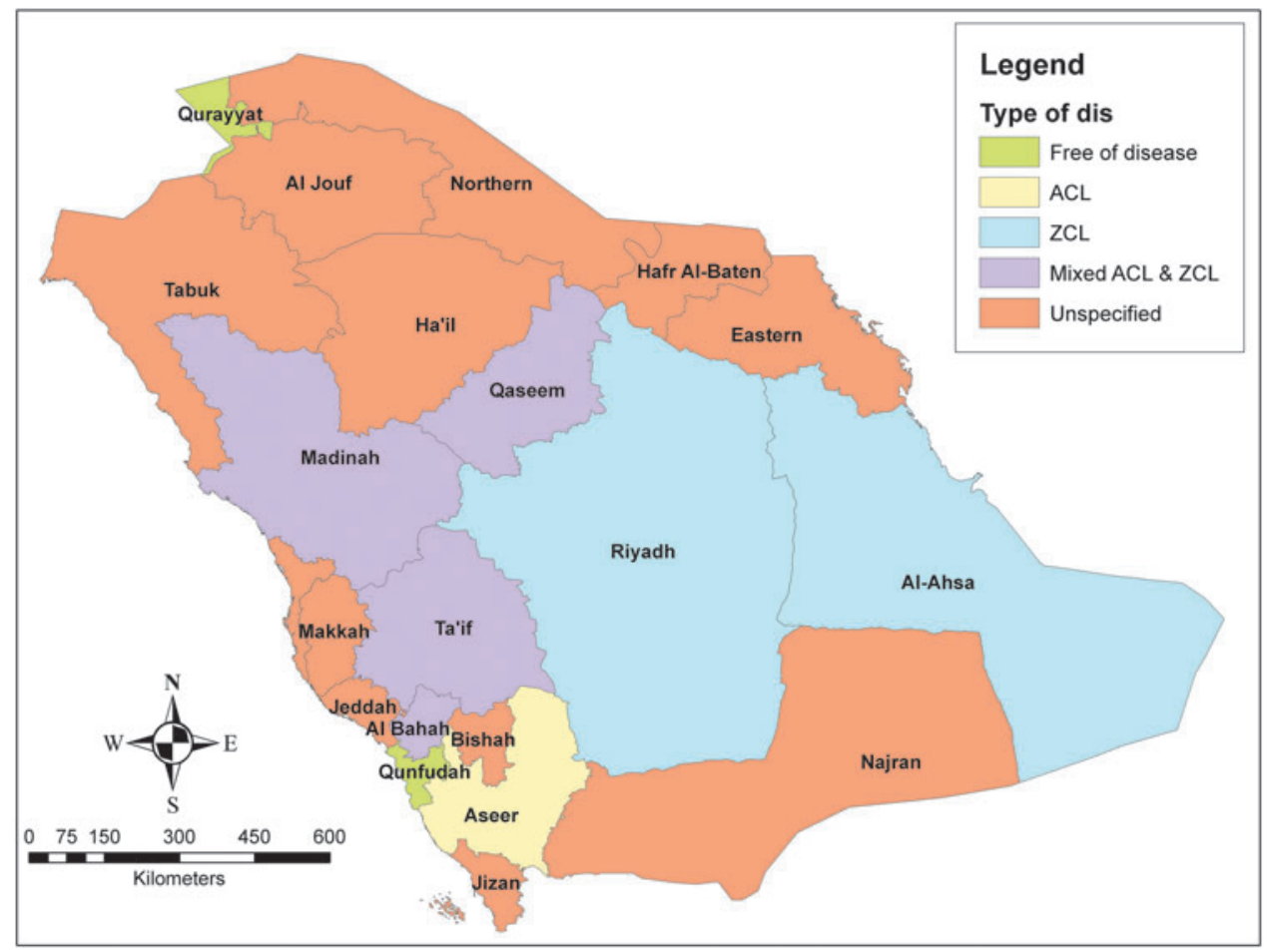

species in charge for the causation of CL in KSA. L. major has been identified as the incriminated species in Riyadh and Al-Hassa regions and appears to be widely distributed in the Kingdom, and by isoenzyme characterization, L. major zymodeme LON-4 has been demonstrated as the typical KSA form of the parasite (Peters et al. 1985). L. tropica has been defined as the main species responsible for CL in Aseer and Al-Bahah provinces, southwest of the country (Al-Zahrani et al. 1989). Several zymodemes of L. tropica have been identified, including LON-71, LON-72, LON-73, LON-10, LON-63, and LON-22 (Peters et al. 1985, Al-Zahrani et al. 1989). Based on these findings and together with the abundance of Phlebotomus papatasi (L. major vector) in many regions, it was thought that $L$. major is involved in most of the country's regions and L. tropica is only incriminated in the southwestern zone (WHO 2007). In contradiction to that suggestion, many recent studies have demonstrated that $L$. tropica is also scattered outside the southwestern area. Incrimination of both L. major and L. tropica in causation of CL in the same region has been demonstrated in many regions, including Al-Madinah, Al-Qaseem, Al-Bahah, and Ta'if (Fig. 4) (Morsy et al. 1991, Shalaby et al. 2011, El-Beshbishy et al. 2013a, Khan and Zakai 2014).

\section{Incriminated Leishmania Vectors in KSA}

Globally more than 90 sand fly species are proven or suspected vectors of Leishmaniasis from over 800 species of sand fly are recorded (WHO 2010). Although about nine species of Phlebotomus have been recognized including: $P$. papatasi, $P$. bergeroti, $P$. alexandri, $P$. kazeruni, $P$. sergenti, $P$. orientalis, $P$. arabicus, $P$. saevus and $P$. major, only five species have been considered or proven to be incriminated as vectors of CL in KSA (Table 2) (Buttlker 1979, Lewis and Büttiker 1982, Ibrahim and Abdoon 2005, Alahmed et al.
2010, Doha and Samy 2010). Of these, P. papatasi is the major and most predominant leishmaniasis vector species.

Killick-Kendrick (1990) has proved that P. papatasi is the main vector of zoonotic cutaneous leishmaniasis (ZCL) in Al-Hassa oasis and probably in other ecologically similar foci in the Kingdom. He detected promastigote stages in the midgut of $P$. papatasi females and by isoenzyme electrophoresis typing were identified to be L. major (zymodeme LON-4), typical to that isolated from human patients. Also in Riyadh region, promastigote stages of Leishmania parasites were isolated from the foregut of $P$. papatasi (Morsy and al Seghayer 1992). Near Abha City, in the highlands of southwest Saudi Arabia (Aseer region), dissected P. sergenti females revealed the presence of flagellates in their midguts, and isoenzyme and monoclonal antibody typing confirmed that these organisms were $L$. tropica corresponding to zymodeme LON-10 and LON-71 and both of them were isolated from human patients from the same locality (Al-Zahrani et al. 1988, 1989). Recently, in Al-Madinah Al-Munawarah province (Madinah), using

Table 2. Distribution of Sand Flies in Kingdom of Saudi Arabia by Region

\begin{tabular}{|c|c|}
\hline $\begin{array}{l}\text { Phlebotomus } \\
\text { species (P.) }\end{array}$ & Region \\
\hline P. papatasi & $\begin{array}{l}\text { Riyadh, Eastern, Northern, Ha'il, Al-Qaseem, } \\
\text { Al-Madinah, Makkah, Al-Jouf, Tabuk, } \\
\text { Jeddah, Al-Ta'if, Al-Hassa, Hafr- Al-Baten, } \\
\text { Bishah. }\end{array}$ \\
\hline P. sergenti & $\begin{array}{l}\text { Al-Madinah, Makkah, Najran, Aseer, Jizan, } \\
\text { Jeddah, Al-Ta'if, Bishah, Al-Bahah. }\end{array}$ \\
\hline P. bergeroti & $\begin{array}{l}\text { Al-Madinah, Makkah, Najran, Aseer, Jizan, } \\
\text { Jeddah, Al-Ta'if, Bishah, Al-Bahah. }\end{array}$ \\
\hline P. arabicus & Najran, Aseer, Jizan, Al-Ta'if, Al-Bahah. \\
\hline No data & \\
\hline
\end{tabular}


semi-nested and conventional PCR protocol, more than $31 \%$ of the collected female $P$. sergenti were found to be infected with $L$. tropica, while $L$. major has been identified in $23 \%$ of the trapped female $P$. papatasi (El-Beshbishy et al. 2013b). However, the actual role of other dominant species of Phlebotomus, for example, bergeroti, arabicus, and alexandri, in CL disease transmission in KSA has not yet been clarified (Table 2).

$P$. papatasi as the most predominant vector of CL occupies, majorly, the Central, Eastern, and Northern regions; however, some sporadic surveys proved their presence in other regions of the Kingdom (Fig. 5) (Alahmed et al. 2010, Doha and Samy 2010). Addressing the seasonal abundance of P. papatasi, a study was carried out in the Central Region of Saudi Arabia (Morsy et al. 1995). It was found that the greatest number of sand flies occurred most commonly during the summer season with peaks in June and another one in September. During winter season, no insect was found and the population density started to appear again from March. The maximum daily indoor activity of $P$. papatasi was found in May, with the largest density time of Phlebotomus detected from 10 to $11 \mathrm{pm}$, while the maximum daily outdoor activity was found in June, with the highest number from 7 to $9 \mathrm{pm}$.

The bioclimatic pattern of $P$. sergenti, the second most important vector species (Fig. 6), has been addressed in AlBahah region (Doha and Samy 2010). It has been found that $P$. sergenti is present at all altitudes. Concerning the seasonal activity, it has been shown that $P$. sergenti is available throughout the year with a peak in density during May and June and then population dropped markedly in November. In addition, this species tend to prefer outdoor habitats. Different species of sand flies show different distribution pattern and seasonal variation, but in general, they survive at temperatures above $16^{\circ} \mathrm{C}$, up to $44^{\circ} \mathrm{C}$, and are mostly found between the months of May and November, showing maximum activity on warm clear nights with low wind speed
(Salam et al. 2014). Certain species, like $P$. bergeroti, $P$. papatasi, and $P$. arabicus, prefer indoor habitats, while others, like $P$. alexandri, are found in outside environment (Doha and Samy 2010).

\section{Leishmania Reservoirs in Saudi Arabia}

Of the two species of Leishmania parasites of human, only L. major has been isolated from animal reservoirs. Several animal species have been identified as the principal reservoirs for L. major. Of the five species of rodents caught in Northern and Western Saudi Arabia including: Meriones libycus, Psammomys obesus, Rattus rattus, jaculus, and Hystrix indica, the first species was the most abundant (90\%), and the isoenzyme electrophoresis identification of the Leishmania isolates from both human patients and rodents showed an identical species (zymodeme LON-4) (Bin et al. 2010). In AlHassa oasis and Al-Madinah Al-Munawarah and Al-Qaseem provinces, the desert rodents, Psammomys obesus and $M$. libycus, are the main reservoir hosts (Elbihari et al. 1984, Peters and Al-Zahrani 1987, Al-Tawfiq and AbuKhamsin 2004, El-Beshbishy et al. 2013b).

It was observed by the authors during their work on leishmaniasis control program that a complete cycle of Leishmania parasite transmission is running in some plains at the periphery of cities among populations of rodents (Psammomys obesus) by the efficient sand fly vector $(P$. papatasi), where the rodents provide the bloodmeals and their burrows provide shelters for them to breed. When humans come closer or inhabit these plains, they become accidently bitten by infected sand flies and develop ZCL. The fat-tailed sand rat, Psammomys obesus, population colonizes these open lands and rely mainly on rimth plant (Haloxylon salicornicum) for feeding and make their burrows underneath these shrubs. When new buildings are to be constructed at

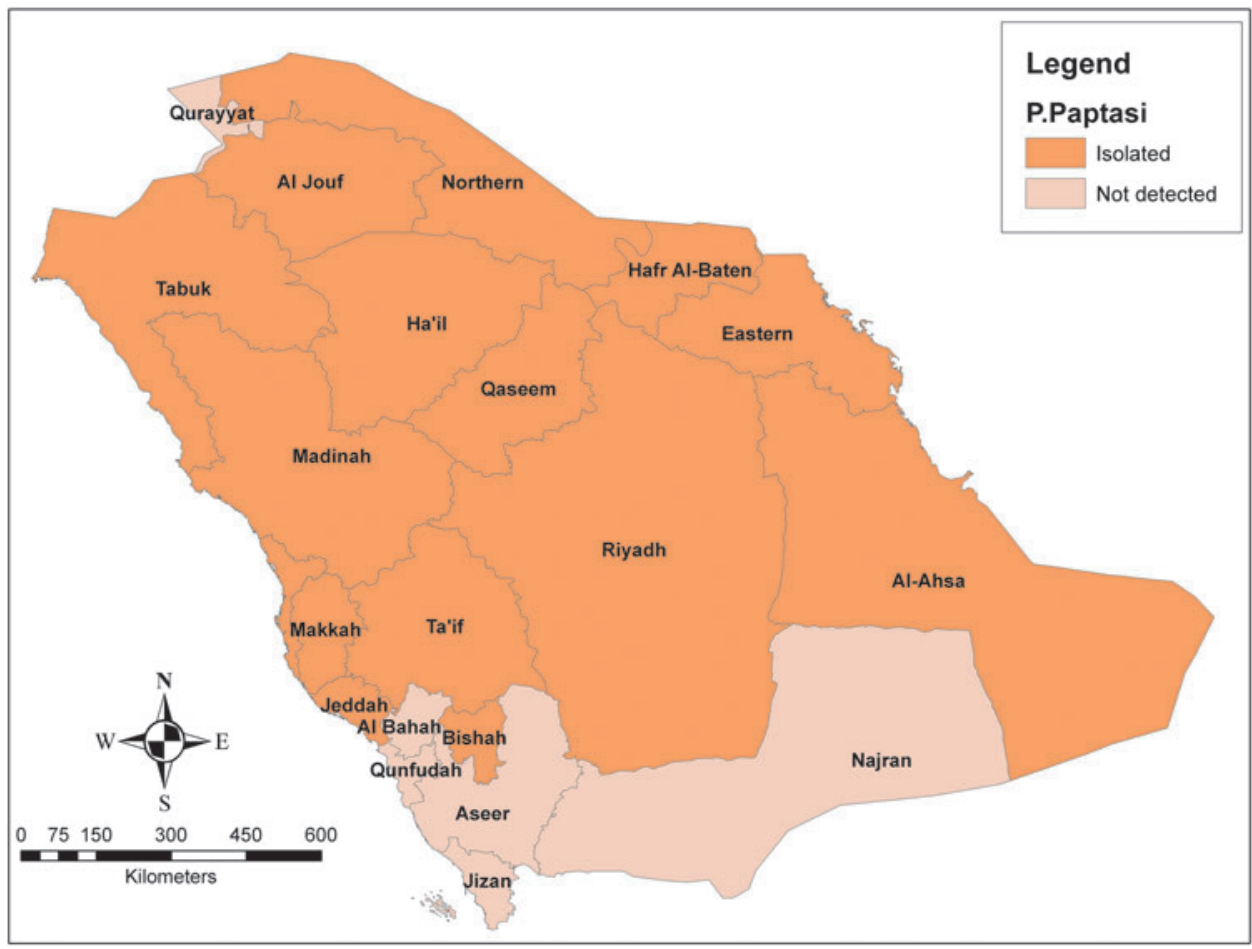

FIG. 5. Geographical distribution of Phlebotomus papatasi. 
FIG. 6. Geographical distribution of Phlebotomus Sergenti.

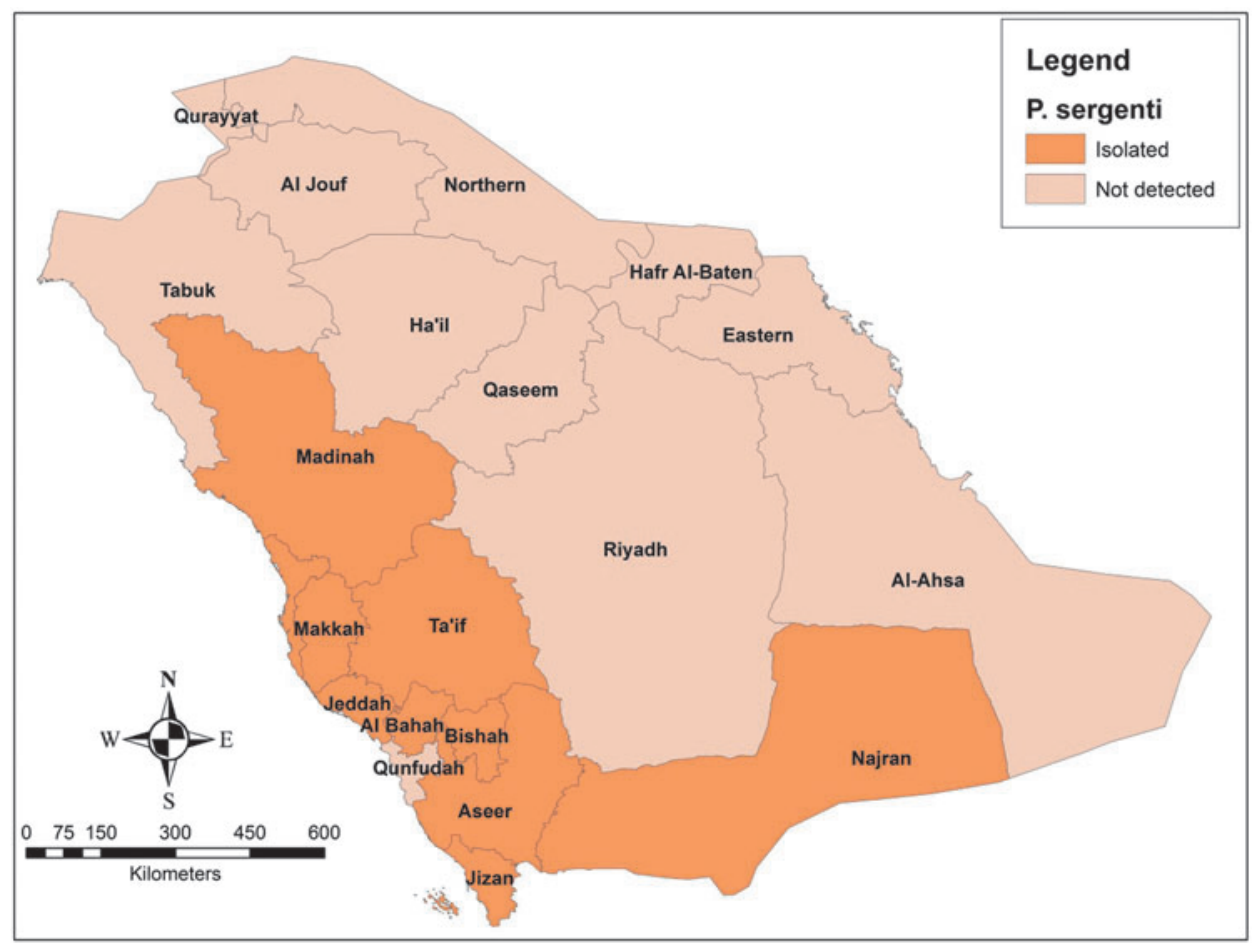

closer neighborhoods or villages, labors and other inhabitants become victims for leishmaniasis; consequently, many cases start to appear.

\section{Clinical features}

Following inoculation of promastigotes into the skin by a sand fly, it takes several weeks to months for the lesion(s) to appear; the incubation periods for $L$. major and L. tropica are less than 4 and 2-8 months, respectively (WHO 2010). Typically, the lesion(s) start as a raised papule, over several weeks it turns to a nodule or a plaque, with ulceration and crust formation occurring at the center of the lesion(s). Satellite lesions, which are characterized by the presence of small papules at the edges of the lesion, are reported to occur with $L$. major infections (Fig. 7). Typically, CL affects the

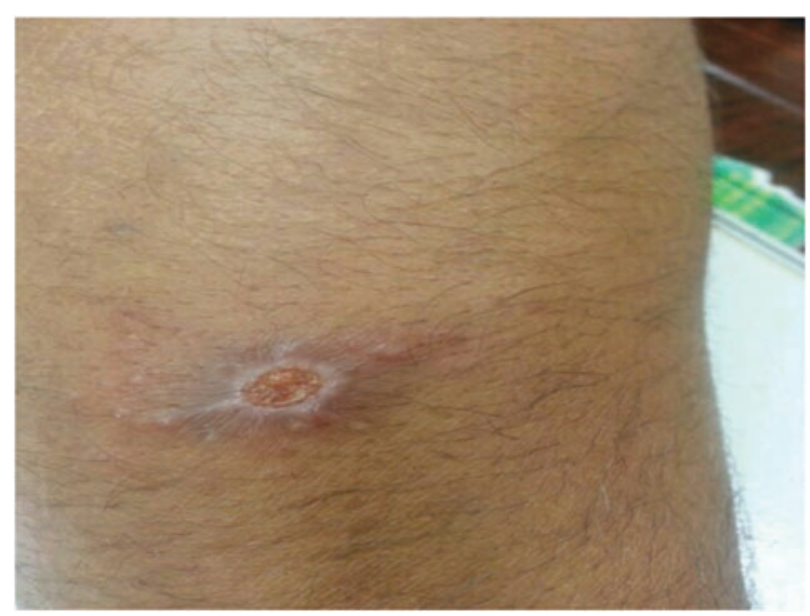

FIG. 7. Satellite papules of CL. exposed parts of the body; the face, neck, arms, and legs are the commonly affected sites. Moreover, lesions are painless, unless secondarily infected, and the subject may present with a single or multiple lesions.

All the data, in KSA, describing the demographic characteristics and clinical presentation of patients are hospital based, and to the best of our knowledge, so far there is no community based literature. According to the $\mathrm{MoH}$ data for the year 2015, males were affected more than females with the percentages of $78 \%$ and $22 \%$, respectively. This could be explained by the Islamic catechistic where the ladies cover their whole body by wearing Hijab. However, males are more exposed to sand flies because of their behavior and occupations. With regards to the nationality of patients, there was no remarkable difference between Saudis and expatriates, $52.4 \%$ were Saudis and $47.6 \%$ were non-Saudis. However, the majority of the affected expatriates were construction workers and laborers working in farms and the fields (Fig. 8a, b). The affection of the Saudis was most probably related to their behavior of spending night(s) at the periphery of towns, where the flies are abundant. Concerning the age distribution, teenagers and adults (15-44 years) were the most commonly affected age category, representing $62.8 \%$ of the total affected population. Generally, these demographic characteristics are the same over the preceding years with minor variation in the obtained figures. In addition, the demographic features of CL patients as per regional assessments did not differ from the overall cumulative data (studies from Al-Hassa, Ta'if, and Hail regions) (Amin et al. 2013, Khan and Zakai 2014, Haouas et al. 2015).

The clinical presentation of CL can be variable. Solitary skin lesion is the most frequent; in a study of 607 participants carried out in the eastern part of KSA, 71\% presented with a single lesion (Al-Tawfiq and AbuKhamsin 2004). Another small study conducted in Ta'if also showed an occurrence of single lesion in $81 \%$ of participants (Khan and Zakai 2014). 
a

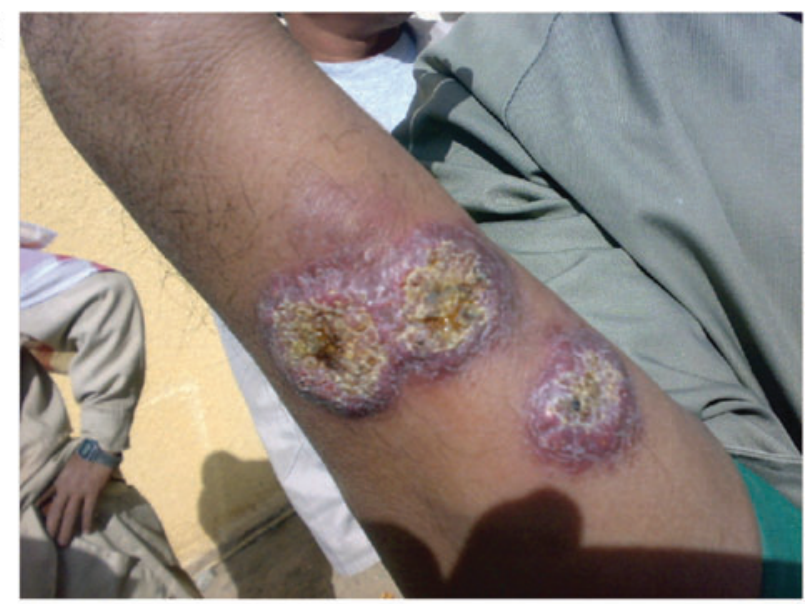

b

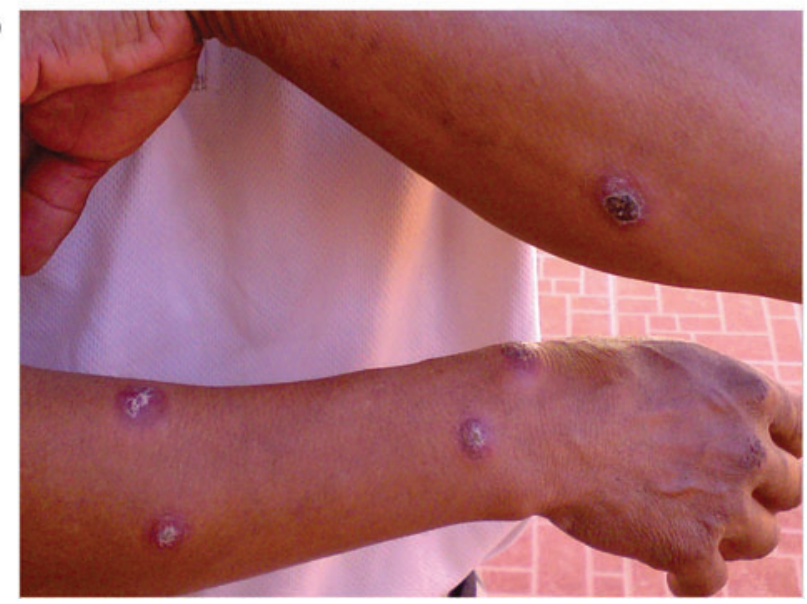

FIG. 8. (a, b) Two expatriates work in the same farm presenting with multiple lesions.

However, it seems that multiple lesions were also not uncommon, in an evaluation of 197 patients; none of them presented with a single lesion (El-Beshbishy et al. 2013a). Overall, CL with multiple lesions tends to affect the nonSaudis who are most probably nonimmune (Fig. 8a, b). However, the disease also tends to be more severe among the nonindigenous of endemic zones, including both non-Saudi and Saudi's living in disease-free zones (Bienzle et al. 1978, Al-Gindan et al. 1984, Kubeyinje et al. 1997, Amin et al. 2013). As for the lesion's site, the most common site affected was the face, followed by the upper limbs, then the lower limbs (Shalaby et al. 2011, Amin et al. 2013, El-Beshbishy et al. 2013a, Khan and Zakai 2014). Morphologically, patients present with different lesion forms, the most common pattern is ulcerative lesions $(90 \%)$, followed by nodular and then nodulo-ulcerative lesions (Al-Tawfiq and AbuKhamsin 2004). Moreover, the lesions' size varies; smaller lesions (1$2 \mathrm{~mm}$ ) are more frequent (El-Beshbishy et al. 2013a).

Disseminated lesions in the form of subcutaneous nodules, satellite papules, and subcutaneous indurations have been reported in some cases, and the potential to disseminate was higher among non-Saudis (Al-Qurashi et al. 2000). This can be linked to the development of herd immunity following previous and recurrent exposures to sand fly bites in disease endemic foci. Enlargement of regional lymph nodes also has been reported to occur in $10 \%$ of cases, and the dissemina- tion through lymphatics may produce subcutaneous nodules connected with palpable thickened lymphatics; this is called sporotrichoid CL, also has been reported to occur among KSA patients (Al-Gindan et al. 1989, Gaafar et al. 1994). Other uncommon clinical forms, including chiclero ulcer, where the lesion involves the rim of the pinna, and mycetoma-like lesions also have been reported in the country (Kubba et al. 1983).

\section{Diagnosis}

A definite diagnosis of CL is made through the combination of clinical history, epidemiological data, and laboratory confirmation. Typical history includes appearance of skin lesion(s) on the exposed parts of the body and endemicity of the region where the patient lives or visited months before the appearance of the lesion(s). In a study, involving 475 participants from KSA, 84\% patients presented with lesions on an exposed body site (Kubba et al. 1987). Generally, neither fever nor systemic manifestations occur with CL. Laboratory confirmation is a crucial element for diagnosis of CL. First, CL has many differentials; several skin conditions manifest in the same manner like CL. A list of 44 clinical conditions was developed by Kubba et al. (1987) as a differential diagnoses of CL. Second, it is unethical to expose patients to toxic, painful, and costly drugs, namely pentavalent drugs, without a confirmed diagnosis.

Many diagnostic tools with huge variations in diagnostic accuracy have been developed for the diagnosis of CL (Goto and Lindoso 2010). Parasitology is still the mainstay for diagnosis of CL due its high specificity. The sensitivity of parasitological tests used varies depending on the geographical location, species, and stage of the lesion; therefore, multiple tests should be performed for each patient if needed (WHO 2010). Specimens are obtained by scrubbing, fine needle aspiration, or biopsy. It is found that fine needle aspiration is superior to scrubbing methods, with a significant difference in the capability of detecting the parasite (Hosseinzadeh et al. 2012). Biopsy has the advantage when the parasites are scanty and abundant tissue is needed. The collected samples undergo direct microscopic examination, culture, or molecular biology. Direct microscopy, searching for the old-world species, has a low sensitivity of $50-70 \%$ as reported by Goto and Lindoso (2010). Culture and molecular diagnosis improves sensitivity and allows identification of species and should be done whenever possible, but their use is limited because of the required infrastructure and technical expertise (Goto and Lindoso 2010, WHO 2010). The recently developed mini- and microculture techniques are easy, less in cost, and more sensitive even when the parasites are scanty, but the microculture does not allow for Leishmania typing (Boggild et al. 2008, de Vries et al. 2015). However, PCR techniques have $100 \%$ specificity and $92.5 \%$ sensitivity in diagnosis of CL in the old world (Al-Hucheimi et al. 2009). Local evaluation, in a study conducted in Al-Madinah region, revealed that PCR technique has a sensitivity of $90.7 \%$, while the sensitivities reported for in vitro culture and microscopy are too low (El-Beshbishy et al. 2013a). Serology is, generally, of no value in diagnosis of CL. Overall, there is an urgent need for an easy to use test for diagnosis of CL, particularly in the field and remote areas.

As for the current practice of diagnosing CL in KSA, most of the healthcare providers and dermatologists rely only on 
clinical presentation and epidemiological data. This might be due to the low sensitivity of microscopy, the requirements of skilled personnel to handle the procedures, and the technical demands of available diagnostic tools. However, the lack of clear national guidelines that compel the health provider to make use of proper diagnostics also has contributed to this malpractice. Furthermore, the available diagnostics have not yet been evaluated in the country. A message addressing the significance of confirming diagnosis by laboratory tests needs to be spread to healthcare providers. Nevertheless, evidencebased guidelines and diagnostic algorithm following regional assessment of current tools are advisable. It is known that Leishmania species vary in their response to available therapies; this makes species identification critical to achieve better outcomes of treatment (Hodiamont et al. 2014). KSA is hosting different Leishmania species; a treatment algorithm should involve species determination to aid in selection of the best treatment choice.

\section{Treatment}

OWCL is generally a self-limited disease, with spontaneous healing occurring over months to years. Therapeutic interventions are most often used to accelerate the healing process and to narrow the period of transmission, since infected patients act as source of parasites. In addition, the long time taken for spontaneous healing to occur make the lesion susceptible to secondary bacterial infections and may be associated with disfigurement of the affected part of the body. These circumstances support the need for therapeutic intervention. However, there is no evidence that early interventions prevent scar formation. CL due to $L$. major may heal spontaneously within 4-6 months in $50-75 \%$ of cases and almost in all patients at 8 months, while in L. tropica spontaneous healing occurs within 1 year or longer (WHO 2010, WHO EMRO 2014). In-line with this information, it has been shown, from endemic areas for L. major, that $92 \%$ of previously positive smears have turned to negative at 3.5 months without any therapeutic intervention (Zakraoui et al. 1995).

Therapeutics for CL consist of local/topical (paromomycin ointment, imidazole ointment, local infiltration of lesion with antimonials), systemic (antimonials, azoles, miltefosine, amphotericin B and its formulations), and physical (e.g., thermotherapy, cryotherapy) interventions. The efficacy profile of these therapies varies depending on the type of therapy, the causing Leishmania species, and the geographical regions (González 2013, Reveiz et al. 2013).

Ideally, the criteria for selection of the best therapy must include the clinical presentation, the identity of the isolated organism, and the regional evidence-based experience. CL can be classified based on the clinical presentation into mild, moderate and severe forms. Using the WHO criteria of CL case definitions, a mild CL can be described as few (fewer than four) lesions, small in size $(<4 \mathrm{~cm})$, and not potentially disfiguring (e.g., not on the face); L. major is the causative organism, and the patient is not immunocompromised or suffering from chronic illnesses. Incrimination of $L$. tropica (or $L$. infantum) instead of L. major, in the presence of other mild criteria, turns the classification into moderate type. However, severe cases present with multiple lesions (four or more), or a big lesion(s) ( $\geq 4 \mathrm{~cm}$ ), or lesions on sensitive and compromised areas (e.g., lips, eyes), or the patient is immunocom- promised or suffering from chronic illnesses. Following the WHO guidelines, apart from the conservative management in the form of cleaning of wounds and use of antibiotic when there is bacterial superinfection, which is applied to all CL patients, mild cases of CL require no therapeutic intervention (wait and see policy), local and physical interventions are administered to treat deteriorating mild cases and moderate ones, and the systemic medicines are preserved for severe or unresponsive lesions to local and physical therapies (WHO EMRO 2014). Screening of patients for contraindications is a prerequisite before commencing systemic treatments.

Clinical trials and experiences on CL treatment, in KSA, are generally scarce; many therapeutic modalities have not been evaluated in longitudinal trials, and most of the conducted studies only focused on Azole efficacy profile in vitro and in vivo. As far as our knowledge, Paromomycin ointment has not been evaluated or studied in KSA, although paromomycin efficacy has been described in many trials since 1990 s in the old world (El-Safi et al. 1990). However, a randomized double-blind trial was carried out to compare the efficacy of two topical drugs: clotrimazole and miconazole creams; 151 lesions distributed among 54 participants were treated for 4 weeks (30 days), only $\sim 16 \%$ of the lesions healed completely in the clotrimazole arm, while none of the lesions treated with miconazole showed complete healing (Larbi et al. 1995). Intralesional pentavalent antimonial therapies have been evaluated and high cure rates were obtained, but the associated pain which sometimes requires local anesthesia, other local side effects, and the need to frequently visit the health facilities may negate recommending this therapy to be in the forefront of the preferred therapeutic list. In two different studies, intralesional antimonials administered on alternate days accomplished cure rates (complete and partial) of $88 \%$ and $97 \%$ (Tallab et al. 1996, Alkhawajah et al. 1997). However, in a small study, aged more than 30 years and of 30 subjects, a $100 \%$ cure was obtained by application of cryotherapy, using $\mathrm{Co} 2$ cryo machine, within 4-5 weeks without remarkable scarring (Bassiouny et al. 1982). Investigating systemic antimony, a dosage of $15 \mathrm{mg} / \mathrm{kg}$ administered parentally over 2 weeks (a total of 12 injections) was used to treat CL, $84 \%$ of the treated lesions achieved the intended outcome at 30 days $(68 \%$ had healed completely and $16 \%$ had improved) (Alkhawajah et al. 1997). However, in a large-scale revision of existing data to address the epidemiology of CL over 42 years in KSA, it was found that parenteral sodium stibogluconate (SSG) at a dose of $20 \mathrm{mg} / \mathrm{kg}$ for 4 weeks had a cure rate of $64 \%$ only (239/373 patients) (Al-Tawfiq and AbuKhamsin 2004). Currently, the WHO recommended regimen of systemic antimonials for CL is $20 \mathrm{mg} / \mathrm{kg}$ for 3 weeks (WHO EMRO 2014). Due to the local findings, this regimen needs to be evaluated carefully in large-scale clinical trials. Furthermore, antifungal oral drugs, including fluconazole, itraconazole, and terbinafine, have been evaluated experimentally and in humans in KSA (Zakai and Zimmo 2000, Alrajhi et al. 2002, Zakai et al. 2003, Khan and Zakai 2014). Overall, good results were obtained with these antifungals, making them a suitable alternative for the other toxic and technically cumbersome regimens.

Due to the variation in efficacy of different regimens among different regions globally, local experience following standard clinical trials is the best way to select the most 
effective therapy. Generally, monotherapies against CL have shown great variation, and drug resistance is an emerging disaster facing the control of leishmaniases (Croft et al. 2006). Exploration of new medicines and development of combination therapies from the existing interventions represent the way forward to treat CL.

With regards to the current practice of treating CL cases in KSA, systemic SSG is superior to other modalities. Antimonials are being used for the treatment of leishmaniases since many decades with successful outcomes, and they are still the first choice for CL treatment in many countries. Unfortunately, in KSA, systemic antimonials are administered to treat CL irrespective of the clinical pattern and the type of causative species. Due to the lack of evidence-based experiences and the absence of clear national guidelines, selection of the appropriate therapy mainly relies on treating physician experience and opinion, and consequently, no consensus on how to treat is expected among dermatologists and health practitioners. A questionnaire-based survey was run to assess the treatment trend of CL in the country, and it has been shown that there was no consistent method used in treating CL, and many disparities were detected (Al-Jaser 2005). Urgent adoption of national CL management guidelines, based on the combination of local experience and international recommendations, is demanded. In addition, clinical trials should be encouraged.

\section{Conclusion}

In summary, KSA is still considered endemic for CL; more efforts and integrated approaches are needed to combat this disease. To handle the potential risk factors, it is advisable for the urbanization process and construction of new buildings to be preceded by some ecological and biological assessments to avoid as much as possible the invasion of disease natural habitats and to apply some control activities if needed. However, vector control activities and health education on use of personal protection measures should be carried out regularly during Hajj pilgrimage to prevent many vector borne diseases, including CL. The disease is endemic in many parts of the country; in some regions neither the causative species nor the incriminated sand fly was identified. Therefore, scientific research should be encouraged to address all aspects of the disease. Actually, identification of the involved parasite species has a major role in decision making concerning management of patients. However, regular evaluation and assessment of flies density and distribution, with the assessment of their behavior, is an integral element for the designing of vector control strategies and proper allocation of resources. Furthermore, the current management practices regarding the diagnosis and treatment of cases need to be modified. Diagnosis of cases through the demonstration of Leishmania tissue stage (diagnostic stage) using laboratory techniques should be encouraged. Nevertheless, development of national diagnostic and management guidelines and algorithms is highly demanded. These guidelines, preferably, should follow local assessments of performances of available diagnostic tools and therapies. Well-designed trials and observational studies in compliance with international standards are the best way to improve the practice of CL handling locally, as well as globally.

\section{Author Disclosure Statement}

No competing financial interests exist.

\section{References}

Alahmed AM, Kheir SM, Al Khereji MA. Distribution of Sandflies (Diptera: Psychodidae) in Saudi Arabia. Res Bult Food Sci Agric Res Center King Saud Univ 2010; 171:5-23.

Al-Gindan Y, Abdul-Aziz O, Kubba R. Cutaneous leishmaniasis in Al-Hassa, Saudi Arabia. Int J Dermatol 1984; 23:194-197.

Al-Gindan Y, Kubba R, el-Hassan AM, Omer AH, et al. Dissemination in cutaneous leishmaniasis. 3. Lymph node involvement. Int J Dermatol 1989; 28:248-254.

Al-Hucheimi SN, Sultan BA, Al-Dhalimi MA. A comparative study of the diagnosis of Old World cutaneous leishmaniasis in Iraq by polymerase chain reaction and microbiologic and histopathologic methods. Int J Dermatol 2009; 48:404-408.

Al-Jaser MH. Treatment trends of cutaneous leishmaniasis in Saudi Arabia. Saudi Med J 2005; 26:1220-1224.

Alkhawajah AM, Larbi E, al-Gindan Y, Abahussein A, et al. Treatment of cutaneous leishmaniasis with antimony: Intramuscular versus intralesional administration. Ann Trop Med Parasitol 1997; 91:899-905.

Al-Qurashi AR, Ghandour AM, Osman M, Al-Juma M. Dissemination in cutaneous leishmaniasis due to Leishmania major in different ethnic groups in Saudi Arabia. Int J Dermatol 2000; 39:832-836.

Alrajhi AA, Ibrahim EA, De Vol EB, Khairat M, et al. Fluconazole for the treatment of cutaneous leishmaniasis caused by Leishmania major. N Engl J Med 2002; 346:891-895.

Al-Tawfiq JA, Abukhamsin A. Cutaneous leishmaniasis: A 46year study of the epidemiology and clinical features in Saudi Arabia (1956-2002). Int J Infect Dis 2004; 8:244-250.

Alvar J, Vélez ID, Bern C, Herrero M, et al. Leishmaniasis worldwide and global estimates of its incidence. PLoS One 2012; 7:e35671.

Alvar J, Yactayo S, Bern C. Leishmaniasis and poverty. Trends Parasitol 2006; 22:552-557.

Al-Zahrani MA, Peters W, Evans DA, Chin C, et al. Phlebotomus sergenti, a vector of Leishmania tropica in Saudi Arabia. Trans R Soc Trop Med Hyg 1988; 82:416.

Al-Zahrani MA, Peters W, Evans DA, Smith V, et al. Leishmania infecting man and wild animals in Saudi Arabia. 6. Cutaneous leishmaniasis of man in the south-west. Trans $\mathrm{R}$ Soc Trop Med Hyg 1989; 83:621-628.

Amin TT, Al-Mohammed HI, Kaliyadan F, Mohammed BS. Cutaneous leishmaniasis in Al Hassa, Saudi Arabia: Epidemiological trends from 2000 to 2010. Asian Pac J Trop Med 2013; 6:667-672.

Bassiouny A, El Meshad M, Talaat M, Kutty K, et al. Cryosurgery in cutaneous leishmaniasis. Br J Dermatol 1982; 107 : 467-474.

Bienzle U, Ebert F, Dietrich M. Cutaneous leishmaniasis in Eastern Saudi Arabia. Epidemiological and clinical features in a nonimmune population living in an endemic area. Tropenmed Parasitol 1978; 29:188-193.

Bin DS, Mostafa OM, Abdoon A, Al-Quraishy SA, et al. Isoenzyme electrophetic characterization of Leishmania major, the causative agent of zoonotic cutaneous Leishmaniasis in North and West Saudi Arabia. J Egypt Soc Parasitol 2010; 40: 465-478.

Boggild AK, Miranda-Verastegui C, Espinosa D, Arevalo J, et al. Optimization of microculture and evaluation of miniculture for the isolation of Leishmania parasites from 
cutaneous lesions in Peru. Am J Trop Med Hyg 2008; 79: 847-852.

Buttlker W. Insects of medical importance in Saudi Arabia. Proc Saudi Biol Sci 1979; 3:239-250.

Copeland HW, Arana BA, Navin TR. Comparison of active and passive case detection of cutaneous leishmaniasis in Guatemala. Am J Trop Med Hyg 1990; 43:257-259.

Croft SL, Sundar S, Fairlamb AH. Drug resistance in leishmaniasis. Clin Microbiol Rev 2006; 19:111-126. Review.

De Vries HJ, Reedijk SH, Schallig HD. Cutaneous leishmaniasis: Recent developments in diagnosis and management. Am J Clin Dermatol 2015; 16:99-109.

Desjeux P. Human leishmaniases: Epidemiology and public health aspects. World Health Stat Q 1992; 45:267-275.

Desjeux P. Information on the epidemiology and control of the leishmaniasis by country and territory. Wholleish/91.30. Geneva: World Health Organization 1991.

Desjeux P. Leishmaniasis. Public health aspects and control. Clin Dermatol 1996; 14:417-423. Review.

Desjeux P. Leishmaniasis: Current situation and new perspectives. Comp Immunol Microbiol Infect Dis 2004; 27:305318.

Doha SA, Samy AM. Bionomics of phlebotomine sand flies (Diptera: Psychodidae) in the province of Al-Baha, Saudi Arabia. Mem Inst Oswaldo Cruz 2010; 105:850-856.

Dye C, Killick-Kendrick R, Ben Ismail R, al-Gindan Y. Zoonotic cutaneous leishmaniasis in Saudi Arabia: Results of a preliminary epidemiological survey in Al-Ahsa oasis. Trans R Soc Trop Med Hyg 1989; 83:493-498.

El-Beshbishy HA, Al-Ali KH, El-Badry AA. Molecular characterization of cutaneous leishmaniasis in Al-Madinah AlMunawarah province, western Saudi Arabia. Int J Infect Dis 2013a; 17:e334-e338.

El-Beshbishy HA, Al-Ali KH, El-Badry AA. Molecular characterization of Leishmania infection in sand flies from Almadinah Al-munawarah province, western Saudi Arabia. Exp Parasitol 2013b; 134:211-215.

Elbihari S, Kawasmeh ZA, Al Naiem AH. Possible reservoir host(s) of zoonotic cutaneous leishmaniasis in Al-Hassa oasis, Saudi Arabia. Ann Trop Med Parasitol 1984; 78:543-545.

El-Safi SH, Murphy AG, Bryceson AD, Neal RA. A doubleblind clinical trial of the treatment of cutaneous leishmaniasis with paromomycin ointment. Trans R Soc Trop Med Hyg 1990; 84:690-691.

Gaafar A, Fadl A, el Kadaro AY, el Hassan MM, et al. Sporotrichoid cutaneous leishmaniasis due to Leishmania major of different zymodemes in the Sudan and Saudi Arabia: A comparative study. Trans R Soc Trop Med Hyg 1994; 88: 552-554.

González U. Cochrane reviews on neglected diseases: The case of cutaneous leishmaniasis. Cochrane Database Syst Rev 2013; 3:ED000055.

Goto H, Lindoso JA. Current diagnosis and treatment of cutaneous and mucocutaneous leishmaniasis. Expert Rev Anti Infect Ther 2010; 8:419-433.

Haouas N, Amer O, Ishankyty A, Alazmi A, et al. Profile and geographical distribution of reported cutaneous leishmaniasis cases in Northwestern Saudi Arabia, from 2010 to 2013. Asian Pac J Trop Med 2015; 8:287-291.

Hodiamont CJ, Kager PA, Bart A, de Vries HJ, et al. Speciesdirected therapy for leishmaniasis in returning travellers: A comprehensive guide. PLoS Negl Trop Dis 2014; 8:e2832.

Hosseinzadeh M, Omidifar N, Lohrasb MH. Use of fine needle aspiration cytology in the diagnosis of cutaneous leishmani- asis: A comparison with the conventional scraping method. Trop Doct 2012; 42:112-113.

Ibrahim AA, Abdoon AM. Distribution and population dynamics of Phlebotomus sand flies (Diptera: Psychodidae) in an endemic area of cutaneous leishmaniasis in Asir Region, Southwestern Saudi Arabia. J Entomol 2005; 2:102-108.

Khan W, Zakai HA. Epidemiology, pathology and treatment of cutaneous leishmaniasis in taif region of Saudi Arabia. Iran J Parasitol 2014; 9:365-373.

Killick-Kendrick R. Phlebotomine vectors of the leishmaniases: A review. Med Vet Entomol 1990; 4:1-24. Review.

Kubba R, Al-Gindan Y, el-Hassan AM, Omer AH. Clinical diagnosis of cutaneous leishmaniasis (oriental sore). J Am Acad Dermatol 1987; 16:1183-1189.

Kubba R, Al Gindan Y, Omer AH, El-Hassan AM. Expanding clinical spectrum of cutaneous leishmaniasis in Saudi Arabia. In: Second Annual Progress Report of the National Leishmaniasis Research Project. Riyadh: Saudi National Center for Science and Technology 1983, pp. 123-143.

Kubeyinje EP, Belagavi CS, Jamil YA. Cutaneous leishmaniasis in expatriates in northern Saudi Arabia. East Afr Med J 1997; 74:249-251.

Lainson R, Shaw JJ. Evolution, classification and geographic distribution. In: Peters $\mathrm{W}$, Killick-Kendrick $\mathrm{R}$, eds. The Leishmaniases in Biology and Medicine, vol. 1. London: Academic Press, 1987:1-120.

Larbi EB, al-Khawajah A, al-Gindan Y, Jain S, et al. A randomized, double-blind, clinical trial of topical clotrimazole versus miconazole for treatment of cutaneous leishmaniasis in the eastern province of Saudi Arabia. Am J Trop Med Hyg 1995; 52:166-168.

Lewis D, Büttiker W. Insects of Saudi Arabia: The taxonomy and distribution of Saudi Arabian Phlebotomus sand flies (Diptera: Psychodidae). Fauna Saudi Arabia 1982; 4:353397.

Morsy TA, Abou el-Ela RG, Rifaat MM, al Dakhil MA. The seasonal and daily activities of Phlebotomus papatasi in Riyadh, Saudi Arabia. J Egypt Soc Parasitol 1995; 25: 699-711.

Morsy TA, al Gahtani YM, Faris RM. Two abnormal cases of anthroponotic cutaneous leishmaniasis in Al Baha, Saudi Arabia. J Egypt Soc Parasitol 1991; 21:675-678.

Morsy TA, al Seghayer SM. A brief note on phlebotomine sandflies in Riyadh, Saudi Arabia. J Egypt Soc Parasitol 1992; 22:437-440.

Morsy TA, Shoura MI. Some aspects of cutaneous leishmaniasis in Riyadh. Saudi Arabia. J Trop Med Hyg 1976; 79: 137-139.

Mosleh IM, Geith E, Natsheh L, Abdul-Dayem M, et al. Cutaneous leishmaniasis in the Jordanian side of the Jordan Valley: Severe under-reporting and consequences on public health management. Trop Med Int Health 2008; 13:855860.

Pearson RD, Sousa AQ. Clinical spectrum of Leishmaniasis. Clin Infect Dis 1996; 22:1-13. Review.

Peters W, Al-Zahrani MA. The leishmaniasis-A common health problem in Saudi Arabia. Saudi Med J 1987; 8: 333-343.

Peters W, Elbihari S, Liu C, Le Blancq SM, et al. Leishmania infecting man and wild animals in Saudi Arabia. 1. General survey. Trans R Soc Trop Med Hyg 1985; 79:831-839.

Reveiz L, Maia-Elkhoury AN, Nicholls RS, Romero GA, et al. Interventions for American cutaneous and mucocutaneous leishmaniasis: A systematic review update. PLoS One 2013; 8:e61843. 
Salam N, Al-Shaqha WM, Azzi A. Leishmaniasis in the Middle East: Incidence and epidemiology. Plos Negl Trop Dis 2014; 8:e3208.

Shalaby I, Gherbawy Y, Jamjoom M, Banaja AE. Genotypic characterization of cutaneous leishmaniasis at $\mathrm{Al}$ Baha and $\mathrm{Al}$ Qasim Provinces (Saudi Arabia). Vector Borne Zoonotic Dis 2011; 11:807-813.

Tallab TM, Bahamdam KA, Mirdad S, Johargi H, et al. Cutaneous leishmaniasis: Schedules for intralesional treatment with sodium stibogluconate. Int J Dermatol 1996; 35:594-597.

WHO. Control of the leishmaniases. World Health Organ Tech Rep Ser 2010;(949): xii-xiii, 1-186.

WHO. Leishmaniasis: Fact sheet on leishmaniasis. Available at: www.who.int/mediacentre/factsheets/fs375/en.

WHO. Report of the consultative meeting on cutaneous leishmaniasis. Geneva, WHO Headquarters, 30 April to 2 May 2007.

WHO EMRO. Cutaneous leishmaniasis in Saudi Arabia| Country | Neglected tropical diseases. Available at: www.emro.who.int/ neglected-tropical-diseases/countries/cl-saudi.html.

WHO EMRO. Manual for case management of cutaneous leishmaniasis in the WHO Eastern Mediterranean region.
WHO Regional Publications, Eastern Mediterranean Series; 35. 2014.

Zakai HA, Zimmo SK. Effects of itraconazole and terbinafine on Leishmania major lesions in BALB/c mice. Ann Trop Med Parasitol 2000; 94:787-791.

Zakai HA, Zimmo skh, Fouad MA. Effect of itraconazole and terbinafine on Leishmania promastigotes. J Egypt Soc Parasitol 2003; 33:97-107.

Zakraoui H, Ben Salah A, Ftaiti A, Marrakchi H, et al. [Spontaneous course of lesions of Leishmania major cutaneous leishmaniasis in Tunisia]. Ann Dermatol Venereol 1995; 122: 405-407.

Address correspondence to: Abuzaid A. Abuzaid Infectious Diseases Control Directorate Ministry of Health King Abdulaziz Street Riyadh 11111 Kingdom of Saudi Arabia

E-mail: abuzaidabdalla@yahoo.com 